\title{
Compression of Feedback for Adaptive Transmission and Scheduling
}

\author{
In multiuser wireless radio systems, it may be possible to increase throughput by \\ reducing the feedback-rate of channel quality data while meeting quality of \\ service requirements.
}

By Thomas ERIKSSON ANd Tony Ottosson

ABSTRACT | For wireless systems with adaptive modulation and/or scheduling, feedback of channel quality information is often necessary. It has been questioned whether the increased system performance is worth the additional feedback rate and the increased algorithm complexity.

In this paper, we study how the feedback rate can be minimized, without losing the gains due to adaptive modulation and multiuser diversity. We present an in-depth study of the literature in the area, and evaluate the performance of several state-of-the-art channel quality feedback schemes. By illustrating the compromise between system throughput and feedback channel rate for various schemes, we are able to give valuable insight in choice of method for feedback rate reduction. A major conclusion is that for multicarrier systems, a lossy compression scheme is the best choice, while for single-carrier systems, schemes limiting feedback to only high-SNR users show good performance. Another conclusion is that there are still many issues to study before the schemes can be used in practice.

KEYWORDS | Adaptive transmission; compression; feedback schemes; multiuser diversity; scheduling

\section{INTRODUCTION}

Diversity, in many forms, can provide considerable advantages for communication over a fading channel. Multiuser diversity, introduced in [1], exploits the fact that in a multiuser environment there is a high probability that,

Manuscript received December 19, 2006; revised April 27, 2007.

The authors are with the Department of Signals and Systems, Chalmers University

of Technology, S-412 96 Göteborg, Sweden (e-mail: thomase@chalmers.se; tonyo@chalmers.se).

Digital Object Identifier: 10.1109/JPROC.2007.907131 in a given radio resource (e.g., a timeslot or a timefrequency bin), at least one of the users experiences a highquality link. To benefit from multiuser diversity, we need to implement a scheduling algorithm that for that radio resource select the user with the best channel condition, with the condition that the given quality-of-service (QoS) requirements should be fulfilled. Once the scheduling algorithm has decided which user should transmit in the radio resource, adaptive transmission is used to maximize the throughput. That is, the transmitter should select the combination of transmit power, modulation, channel coding and automatic repeat request (ARQ) protocol that maximizes the throughput. This strategy increases the attainable throughput and system spectral efficiency with the number of users [2].

In order to choose the modulation and coding scheme (MCS) and prioritize among the users, the scheduler must, in theory, know the channel quality [signal-to-noise ratio (SNR)] of all users at all times. If the mobile terminals estimate (predict) their instantaneous channel quality and feed it back to the base station, the scheduler can use this information to schedule a user that momentarily has an above-average channel quality, and assign a modulation form appropriate for the SNR level. However, the spectrum resource that must be reserved for channel quality feedback can be very large, and may make multiuser diversity difficult to exploit in practice. The problem of SNR feedback is especially challenging in case of orthogonal frequency-division multiplexing (OFDM) transmission over frequency-selective channels [3], where the number of channel gains is directly proportional to the number of subcarriers [4].

In this paper, we study the possibilities to reduce the feedback rate in multiuser systems with adaptive 
modulation and scheduling. We start with a statement of our assumptions and goals in Section II. A main contribution of this paper is the thorough literature review and discussion of various schemes in Section III. The literature review is followed by an experimental comparison of the proposed schemes in Section IV, where the best algorithms are implemented and compared. In Section V, we discuss the remaining challenges to be solved, and we summarize the conclusions in Section VI.

\section{PROBLEM STATEMENT}

We consider a multiuser system, where a single base station schedules the available spectrum resource to the mobile terminals with a time-domain multiplexing (TDM) scheme, optionally extended with frequency-domain multiplexing (FDM).

We will assume that the users communicate their respective channel state information (CSI) to the base station on a (assumed error-free) feedback channel. The CSI can be either an instantaneous SNR value, or it can be the MCS corresponding to the instantaneous SNR.

Our mission is to find ways to minimize the necessary feedback rate (in bits per symbol per user), while maintaining a high average throughput per user. We will judge the quality of the feedback schemes we discuss by several criteria.

- A major requirement is of course that it should use as little system resources as possible, i.e., a low feedback rate per user.

- An equally important requirement is that the delay should be small. The schemes discussed here all require channel prediction, and such predictors only work well when they predict a short time ahead [5].

- It is not a requirement, but it is advantageous, if the feedback is in terms of SNR values instead of modulation type (MCS feedback). The reason is that the scheduler can do a better job with SNR feedback (see Section IV).

- The feedback algorithm should be as insensitive to channel model changes as possible. All model changes may have to be transmitted if the algorithm relies on model knowledge, which causes additional feedback rate.

- The algorithm should be possible to use with various scheduling algorithms. As we will see later in this document, almost all proposed algorithms are evaluated using a max-SNR scheduler, which does not provide enough fairness to be used in practice.

We will mainly focus on single-antenna systems. We briefly discuss the topic of multiple antennas systems [multiple-input-multiple-output(MIMO)] in Section III-D, but for a more thorough discussion the reader is referred to, e.g., [6] or [7].

\section{FEEDBACK RATE REDUCTION:} OVERVIEW AND STATE-OF-THE-ART

In this section, we give a thorough overview of the literature on methods to reduce feedback rate for multiuser systems. The literature can be grouped into four main categories.

- Scalar quantization methods with optimized SNR thresholds.

- Schemes exploiting the fact that only the best user will be allowed to transmit (max-SNR scheduling), and consequently that feedback from other users than the best is unnecessary.

- Schemes using lossy or lossless compression exploiting the properties of the fading process.

- Schemes for feedback of multiple-antenna beamforming vectors.

There are also some reports that do not fit into the above categories, e.g., [8], where schemes to reduce feedback rate for bursty packet data is discussed.

Next, we discuss the literature belonging to each of the four categories, describing the algorithms and highlighting the pros and cons relative the desirable properties as discussed in Section II.

\section{A. Quantization}

A straightforward way to decrease the feedback rate is by quantization of the SNR measurements before feedback transmission. Since the SNR values are real-valued, some quantization must be applied; the question is how few bits can be used with a maintained high total throughput of the multiuser system.

Johansson [9] and Florén et al. [10] are early studies of the effect of feedback quantization. They conclude that 1-bit quantization is good enough in many cases, if the average SNR of each user is known. In their example, a throughput of more than $90 \%$ of the throughput with unquantized feedback is achievable, with a 1-bit quantizer optimized for the correct average user SNR and number of users. The scheme can be used also when the average user SNR is unknown, but some of the performance is lost in this case, and a 2-bit quantizer may be needed to reach 90\%. Johansson extends his scheme to 1-bit quantization with online adapted individual quantization thresholds in [11] and [12], improving fairness and robustness of the scheme.

In [13], Sanayei and Nosratinia come to the same conclusion as above, that 1-bit feedback is sufficient in most cases. They present a theoretical analysis, and show that the achievable throughput for the 1-bit case and the unquantized case grows with the number of users in the same way, with a small capacity loss for the 1-bit quantizer.

Discussion: Direct scalar quantization of the SNR values as proposed above is a simple and straightforward way of reducing the feedback rate. However, the minimum 
achievable rate is 1 bit per SNR value, which is still considered too high in many cases, especially for multicarrier systems with multiple time-frequency chunks. Another possible problem is that the optimal quantization thresholds depend heavily on the number of users in the system, a number that may not be readily available.

\section{B. SNR-Limited Feedback}

With a max-SNR scheduling policy, where the base station transmits exclusively to the user with the highest SNR, it is unnecessary for users with low SNR to send feedback; the probability that such a user is scheduled is very low. This can be exploited to reduce the feedback rate, a fact that many reports have proposed.

In [14], Gesbert and Alouini propose a selective multiuser diversity (SMUD) scheme, by letting the users report CSI only when the channel quality exceeds a predefined threshold. Since a max-SNR scheduler always schedules the user with the best channel, CSI feedback from low-SNR users is unnecessary. If no users have an SNR that exceeds the threshold, a random user is scheduled. When the number of users is large $(K>25)$, the SMUD scheme leads to a remarkable reduction of the average number of users transmitting feedback, with a reduction of the feedback rate down to $10 \%$ of full feedback for the best case, with small or no loss in terms of system capacity. Hassel et al. [15], improves the SMUD scheme by requesting full feedback transmission from all users if no user had a large enough SNR; this guarantees the full multiuser diversity gain, at the cost of some extra feedback compared with original SMUD. The scheme was extended to antenna selection and space-time block coding in multiple-antenna systems by Vicario and Anton-Haro in [16], with similar conclusions.

Hassel et al. [17] develops the SMUD scheme further by by exploiting multiple SNR thresholds used sequentially. The base station first requests feedback from those users with an SNR exceeding the highest threshold. If there are none, the threshold is successively lowered until a user fulfilling the requirement is found. Special attention is given to problems with feedback collisions, when several users reply to the same threshold query, and to scheduling outage with a scheduling deadline, when no feedback is received before a scheduling decision must be made. The reduction in normalized feedback rate is similar to the SMUD scheme. In [18], the same scheme is theoretically studied under slightly more realistic assumptions.

Svedman et al. [19] discuss an OFDM multiuser scenario, where each user sends feedback about the $S$ strongest subcarriers. To reduce the feedback rate, they divide the subcarriers into clusters, and each user computes the average SNR within the clusters and feeds back the indices and SNRs of the $S$ strongest clusters. For each cluster, the base station chooses the user with the highest SNR. With this scheme, some cluster indices may not be fed back by any user, and then the available transmit power is divided only among the assigned clusters. A conclusion is that feedback of only the three strongest clusters gives performance in parity with full feedback.

Hämäläinen and Wichman [20] describes a transmit time selection diversity (TTSD) scheme, where downlink transmission is suspended if the instantaneous received SNR in the mobile station falls below a predefined threshold. TTSD requires only a single bit feedback per user. Further, they show that TTSD outperforms the maxSNR scheduler in case of feedback errors.

In [21], Qin and Berry suggest that in an Aloha-inspired protocol, transmission decisions are individually made by the users based on their SNR, and collisions are resolved later. Their proposed splitting algorithm seeks not only to resolve a collision, but also to find the user with the best channel gain. They show that with the proposed algorithm, the throughput approaches the optimal value as the coherence time of the channel increases.

Discussion: By limiting the feedback to users with high SNR, we have seen that large reduction in feedback rate can be achieved. The proposed methods can be combined with the quantization methods in Section III-A, and possibly also with the compression methods in Section III-C. ${ }^{1}$ A problem is if a scheduler with built-in fairness is used, since then low-SNR users may need to be scheduled, users for which there is no channel information if the thresholds are too high. Another problem is, as in the previous subsection on quantization, that the optimal thresholds are strongly depending on the number of users in the system, and it is not discussed in the literature how to adapt the thresholds in case of rapidly varying number of users. Some of the schemes above can also lead to large scheduling delays.

\section{Data Compression}

Since the channel SNR values are correlated in both time and frequency (depending on the Doppler effect and the delay spread) [4], data compression algorithms exploiting such correlations can substantially reduce the required feedback rate. Lossless compression (Arithmetic coding, Lempel-Ziv, etc.) and lossy compression (transform coding, linear prediction, etc.) are established techniques to realize the compression gains, and some authors have proposed such schemes.

An early paper is [22] by Cheon et al., where correlation in time is exploited, and encoding of differential bit-loading vectors ${ }^{2}$ is proposed.

Lestable and Battelli [23] propose the use of LempelZiv-Welch (LZW) coding [24] of the bit-loading vectors in

\footnotetext{
${ }^{1}$ Note that the combination of an SNR-limited and a compression scheme is not obvious; with an SNR-limited scheme, the base station does not have access to the history of SNR values for all users at all time/ frequency slots, which is a prerequisite for compression methods relying on time and frequency correlations.

${ }^{2}$ The bit-loading vectors describes how the bits should be subdivided over the carriers in a multicarrier system. This corresponds to the choice of modulation format of each carrier, which is a function of the carrier SNR.
} 
a multicarrier transmission system. The best compression, with a feedback rate that is $\sim 25 \%$ of the uncompressed case, is achieved when the terminals are moving at a slow speed.

Nguyen et al. [25] suggest a scheme based on runlength coding [26] of differentially encoded bit-loading vectors. The best performance is again for slowly moving terminals, with a feedback rate of only $10 \%$ of the uncompressed case.

Jimenez et al. [27] use Huffman coding in a MIMOOFDM system to compress the feedback to $50 \%$ of the original size. They also evaluate the effect of errors in the feedback channel and suggest update rates to avoid problems.

Gross et al. [28], [29] assume that in contrast to other reports discussed here, the base station has perfect knowledge of the CSI of each user, and discuss instead the signaling overhead in assigning subcarriers to the users in an orthogonal frequency-division multiple-access (OFDMA) system. They employ a simple differential scheme to reduce the signalling. An interesting part of their work is the maximization of the overall throughput, when the signaling overhead is considered in the optimization process.

In contrast to the lossless schemes above, the scheme proposed by Eriksson and Ottosson in [30] relies on lossy compression of the real-valued SNR values. Using the system proposed in [31], a Discrete Cosine Transform (DCT) is used for decorrelation of the SNR vectors, followed by scalar quantization of optimally bit-allocated transform coefficients. If the terminals are not moving too fast, the quantizer indices can be downsampled, by sending indices only every $N$ th frame. In the studied cases, they are able to reduce the feedback rate to between $2 \%$ (slow terminal speed) and 20\% (fast terminal speed) compared with uncompressed feedback of MCS values.

In the previous section, we described a scheme by Svedman et al. [19], where only the $S$ strongest subcarriers are fed back. This scheme can also be viewed as a simple lossy compression scheme, since the subcarriers are grouped into clusters, thereby reducing the feedback rate at the cost of a decreased accuracy over the cluster.

Discussion: Data compression methods can be very powerful for feedback rate reduction, as shown in Section IV. A requirement is that rather detailed longterm channel and system information must be fed back to the base station, a topic that is not covered in the literature. The schemes in this subsection are often more computationally demanding than the schemes in previous subsections.

\section{Multiple-Antenna Systems}

While the use of multiple antennas significantly impacts the design of the adaptive modulation and scheduling schemes compared with single-antenna systems, a common need is feedback of CSI. The channel information in the multiantenna case consists of complex unit-norm beam-forming vectors [multiple-input-single-output (MISO)] [32], [33] or matrices (MIMO) [34], [35], and of SNR values (or absolute channel gain) to exploit multiuser diversity [2], [36].

In the literature on this topic, the focus lies on feedback of the beam-forming vectors/matrices. Most of the reports employs variations of vector quantization (VQ) (see, e.g., [37] for an introduction to VQ) to solve the feedback quantization issue. Some authors propose random VQ [38]-[40], which has the advantage of performing close to optimal quantization, and being very amenable to analysis. Other authors suggest criteria for optimization, and derive VQ optimization algorithms [32], [41], [42]. In [33] and [43], it is shown that the VQ design problem is related to the problem of Grassmannian line packing. In the case of multiuser multiantenna systems, Kountouris et al. [44] propose that only a small group of users is selected for feedback of full CSI (the group can be selected from rough channel estimates).

Discussion: The literature on feedback for multipleantenna systems is voluminous, and we have only scratched on the surface here. To read more on this topic, see, e.g., [6] and [7], which are tutorials on multiuser MIMO where the issue of feedback of CSI for MIMO is thoroughly discussed.

\section{EXPERIMENTS}

In many cases, it is not possible to directly compare the results presented in the various reports, since the experimental setups are too different for a fair comparison. Therefore, we have implemented many of the algorithms we have discussed in Section III, both for a single-carrier system and for a multicarrier (OFDMA) system to cover most interesting aspects of the feedback schemes. Some adaptation and small changes of the algorithms have been necessary to fit the setup, as described next.

\section{A. Experiments on a Multicarrier System}

We have chosen a multiuser system with adaptive modulation and scheduling as proposed in [31], which is an OFDM system with 512 subcarriers whereof 416 are used for transmission of data. The time-frequency grid is grouped into a set of chunks, where each chunk consists of 8 subcarriers times 12 OFDM symbols. Each user computes the average SNR in each chunk, and feeds back this information to the scheduler. Based on feedback from all users, the scheduler chooses which user to schedule and what modulation to use, for each chunk. There are eight different possible modulation forms, from binary phaseshift keying (BPSK) (1 bit per symbol) up to 256-QAM ( 8 bits per symbol), and the optimal choice of modulation (MCS) is determined from the reported SNR in the respective chunk according to [31]. As a reference system, 
direct MCS feedback at a rate of 3 bits per chunk and user, is used.

We have used the ITU Vehicular A model, with power delay profiles as given in [45]. The mobile speed is $50 \mathrm{~km} / \mathrm{h}$.

In order to make a fair comparison between the different schemes, we have adapted the algorithms slightly to the system under consideration.

- The algorithm in [30] is implemented directly from the paper.

- The lossless compression algorithms in [23] and [25] are not implemented, but the performance results are taken directly from the reports, as accurately as possible adapted to the system model here. Due to differences in the system model between their reports and this, the results are slightly uncertain, but they will still approximately indicate the performance.

- The algorithm in [19] was implemented directly, with a small change; if a time-frequency chunk is unassigned, a random user is assigned to that chunk (using BPSK), instead of dividing the available transmit power among the assigned chunks only as suggested in the paper. The reason is that it is usually necessary to transmit in every chunk all the time, for all mobiles to continuously predict the channel quality. Further, the extra waterfilling gain achieved by the original idea would make all comparisons unfair. Unfortunately, this change had a bad impact on the results from this algorithm, and we decided to exclude the results from the plots.

- The algorithms in [14] and [15] are adapted to the OFDM case by independently applying the algorithms to the chunks. The thresholds are reoptimized for all tested cases. Further, since feedback is only transmitted for a subset of the chunks, we must also send indices of the active chunks, which costs considerable additional feedback.

- The 1-bit quantization scheme suggested in, e.g., [9] and [10] is implemented separately for each chunk of each user. The quantization thresholds are reoptimized for all tested cases.

In Fig. 1, we show the average system throughput (in bits $/ \mathrm{s} / \mathrm{Hz}$ ) as a function of the feedback rate (in bits/ chunk). As references, the throughput using full MCS feedback at 3 bits/chunk, and unquantized SNR feedback are shown. For some methods, it is possible to adjust the feedback rate to the desired value, while others have no such possibility; the latter are thus represented by single marks in the figure.

We see that the method based on lossy compression [30] gives the best compromise between throughput and feedback rate. Note that schemes sending feedback of SNR values instead of MCS can asymptotically reach a higher throughput, since the scheduler can exploit the better accuracy of a real-valued SNR level.

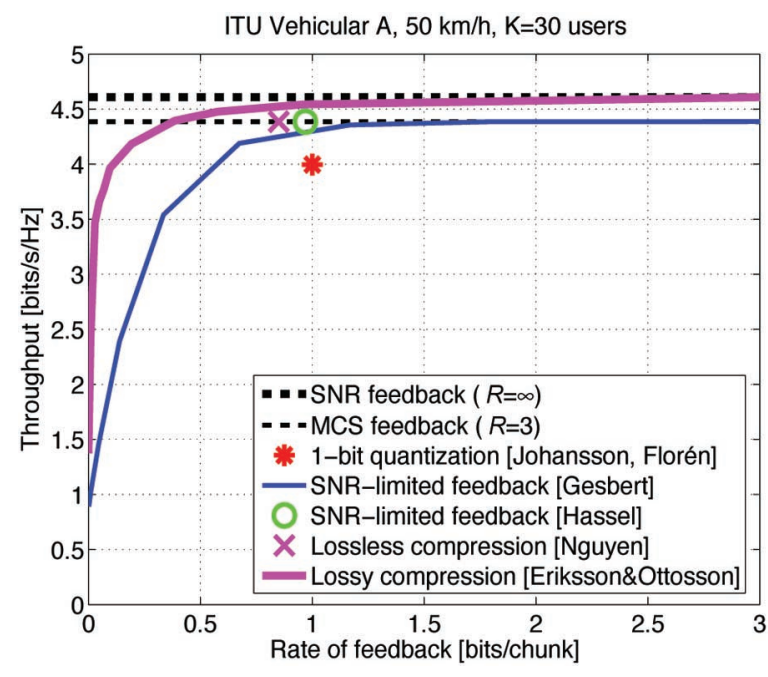

Fig. 1. Throughput as a function of the rate of feedback $R$, for a multicarrier system with 30 users. The thin dashed line indicate the asymptotic performance for the SNR-limited and lossless compression methods, whose performances are upper-bounded by full MCS feedback at 3 bits per chunk, while the thick dashed line indicate the asymptotic performance for lossy compression of SNR values, whose performance is upper-bounded by unquantized SNR feedback (require an infinite rate).

\section{B. Experiments on a Single-Carrier System}

Some of the methods implemented above for a multicarrier system were originally proposed for singlecarrier systems, and to make fair comparisons, we have also implemented a simple single-carrier system.

We assume a single-carrier multiuser system with $N$ users with independent and identically distributed (i.i.d.) flat Rayleigh-fading channels with the same average SNR. The coherence time is set to one packet, which means that the channel quality is (roughly) the same over an entire packet, and that the channel quality is uncorrelated between packets. The users report their SNRs on an error-free feedback channel, and the user with the highest SNR is scheduled. We use adaptive modulation using eight different modulation forms, from BPSK (1 bit per symbol) up to 256-QAM (8 bits per symbol), with the optimal choice of modulation determined from the reported SNR for each user as in the previous subsection.

The schemes in Sections III-A and III-B are compared. The compression methods in Section III-C are not implemented in the single-carrier case, since they mainly rely on correlation between the subcarriers in a multicarrier system.

In Fig. 2, we show the average system throughput (in bits $/ \mathrm{s} / \mathrm{Hz}$ ) as a function of the feedback rate (in bits/ chunk). As references, the throughput using full MCS feedback at 3 bits/chunk, and unquantized SNR feedback, are shown. In this case, we see that the SNR-limited feedback methods works best. 


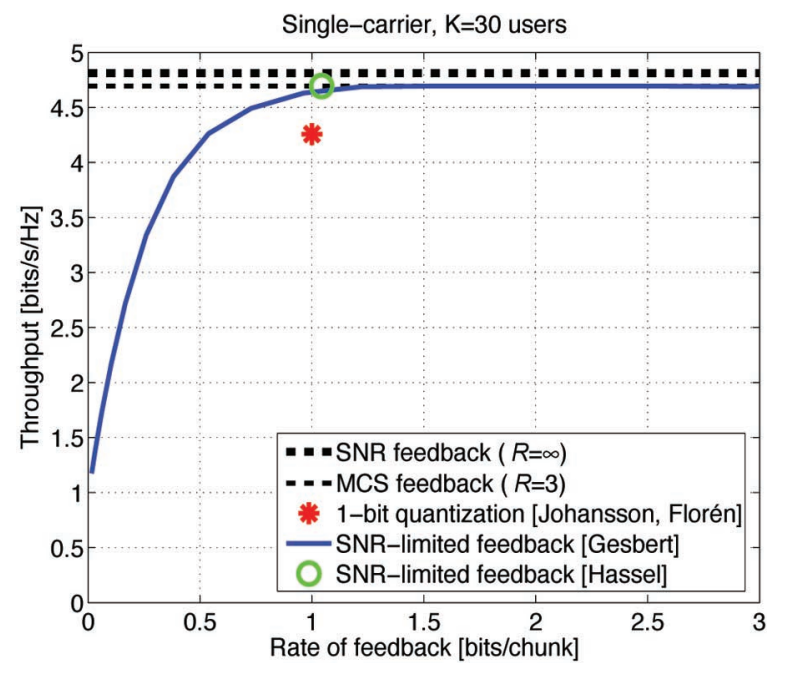

Fig. 2. Throughput as a function of the rate of feedback $R$, for a single-carrier with 30 users. As in Fig. 1, the thin and thick dashed lines indicate asymptotic performances.

In Fig. 3, the throughput as a function of the number of users in a single-carrier system is shown. The simple quantization method works well for a few users, but as could be expected, the SNR-limited methods improve faster with the number of users.

\section{CHALLENGES}

The area of feedback compression for adaptive modulation and scheduling is new, and it is not surprising that there are still issues to be solved before full rollout.

- Scheduling versus feedback compression: Most reports assume a max-SNR scheduler, since it gives the highest multiuser diversity gains. However, since the max-SNR scheduler does not provide any fairness, other schedulers (proportional-fair, fair queueing, exponential rule, etc.) must be considered in practice. The scheduler may have a large impact on the necessary feedback rate; with fair schedulers, feedback cannot, in general, be limited to the user with the highest SNR, which may penalize the algorithms in Section III-B in particular. The topic of joint scheduling and feedback compression is briefly touched in, e.g., [11], [20], and [46], but the area deserves more attention.

- Channel prediction versus feedback compression: All algorithms in this report require some kind of channel prediction for SNR feedback. As described in [5], channel prediction relies on correlation between consecutive channel gains, which is true also for the feedback compression algorithms in Section III-C. Further, most reports assume perfect channel prediction, which is not true in practice [5]. To fully understand the consequences, feed-

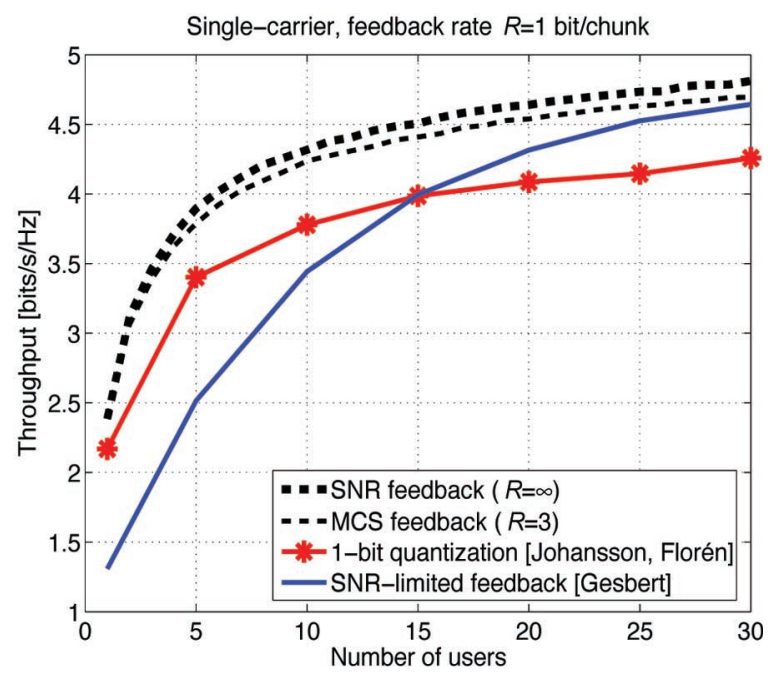

Fig. 3. Throughput as a function of the number of users, for a single-carrier system at an average feedback rate $R$ of 1 bit/chunk for each user. As in Fig. 1, the thin and thick dashed lines indicate asymptotic performances.

back compression and channel prediction should be jointly treated.

- Delay: Some of the proposed schemes have a considerably delay before scheduling can take place. While some reports discuss the delay issue, e.g., [47] and [15], there is a lack of studies discussing the consequences of delay for different feedback compression algorithms.

- Feedback errors: Few reports discuss the effect of feedback errors in connection with feedback compression [20], [27], [48]. Since errors on the feedback channel can potentially destroy most of the multiuser diversity gain, the area deserves more attention.

\section{CONCLUSION}

We have studied the problem of feedback quantization and compression for wireless systems with adaptive modulation and scheduling. A thorough literature study is followed by implementation and comparison of many feedback compression schemes.

A major conclusion is that for multicarrier systems, a lossy compression scheme is by far the best choice. For single-carrier systems, schemes limiting feedback to only high-SNR users show good performance.

From the study in this paper, it seems proper to conclude that the best possible feedback scheme is not invented yet. From the discussion and the experimental results, we can foresee that a combination of a lossy compression scheme and a SNR-limited feedback scheme would be a good candidate for a future feedback compressor. We also conclude that several issues must be addressed before feedback compression can be used in practice. 


\section{REFERENCES}

[1] R. Knopp and P. A. Humblet, "Information capacity and power control in single-cell multiuser communications," in Proc. IEEE Int. Conf. Commun., Seattle, WA, 1995, vol. 1, pp. 331-335.

[2] P. Viswanath, D. N. C. Tse, and R. Laroia, "Opportunistic beamforming using dumb antennas," IEEE Trans. Inform. Theory, vol. 48, no. 6, pp. 1277-1294, 2002.

[3] M. Sternad, T. Ottosson, A. Ahlén, and A. Svensson, "Attaining both coverage and high spectral efficiency with adaptive OFDM downlinks," in Proc. IEEE Veh. Technol. Conf., Orlando, FL, 2003, vol. 4, pp. 2486-2490.

[4] W. Wang, T. Ottosson, M. Sternad, A. Ahlén, and A. Svensson, "Impact of multiuser diversity and channel variability on adaptive OFDM," in Proc. IEEE Veh. Technol. Conf., Fall, Orlando, FL, 2003, pp. 547-551.

[5] A. Duel-Hallen, "Fading channel prediction and estimation for mobile radio adaptive transmission systems," Proc. IEEE, vol. 95, no. 12, pp. 2299-2313, Dec. 2007.

[6] D. J. Love, R. W. Heath, Jr., W. Santipach, and M. L. Honig, "What is the value of limited feedback for MIMO channels?" IEEE Commun. Mag., vol. 42, no. 10, pp. 54-59, Oct. 2004.

[7] D. Gesbert, M. Kountouris, R. W. Heath, Jr., C.-B. Chae, and T. Salzer, "From single user to multiuser communications: Shifting the MIMO paradigm," IEEE Signal Process. Mag., 2007, in press.

[8] M. Döttling, B. Raaf, and J. Michel, "Efficient channel quality feedback schemes for adaptive modulation and coding of packet data," in Proc. IEEE Veh. Technol. Conf., Fall, Los Angeles, CA, 2004, pp. 1243-1247.

[9] M. Johansson, "Benefits of multiuser diversity with limited feedback," in Proc. IEEE Workshop on Signal Processing Advances in Wireless Commun., Rome, Italy, 2003, pp. 155-159.

[10] F. Florén, O. Edfors, and B.-A. Molin, "The effect of feedback quantization on the throughput of a multiuser diversity scheme," in Proc. IEEE Global Telecommun. Conf., San Francisco, CA, 2003, pp. 497-501.

[11] M. Johansson, "Diversity-enhanced equal access-Considerable throughput gains with 1-bit feedback," in Proc. IEEE Workshop on Signal Processing Advances in Wireless Commun., Lisboa, Portugal, 2004, pp. 6-10.

[12] M. Johansson, "On scheduling and adaptive modulation with limited channel feedback," Signals and Systems, Uppsala University, Uppsala, Sweden, Tech. Rep., 2004.

[13] S. Sanayei and A. Nosratinia, "Exploiting multiuser diversity with only 1-bit feedback," in Proc. IEEE Wireless Commun. Netw. Conf., New Orleans, 2005, pp. 978-983.

[14] D. Gesbert and M.-S. Alouini, "How much feedback is multi-user diversity really worth?" in Proc. IEEE Int. Conf. Commun., Paris, France, 2004, pp. 234-238.

[15] V. Hassel, M.-S. Alouini, G. E. Øien, and D. Gesbert, "Rate-optimal multiuser scheduling with reduced feedback load and analysis of delay effects," EURASIP. Wireless Commun. Netw., vol. 2006, pp. 1-7, 2006.

[16] J. L. Vicario and C. Antón-Haro, "Robust exploitation of spatial and multi-user diversity in limited-feedback systems," in Proc. IEEE Int. Conf. Audio, Speech and Signal Processing,
Philadelphia, PA, 2005, vol. 3, pp. 417-420.

[17] V. Hassel, M.-S. Alouini, D. Gesbert, and G. E. Øien, "Exploiting multiuser diversity using multiple feedback thresholds," in Proc. IEEE Veh. Technol. Conf., Spring, Stockholm, Sweden, 2005, vol. 1, pp. 1302-1306.

[18] Y. Al-Harthi, A. Tewfik, and M.-S. Alouini, "Opportunistic scheduling with quantized feedback in wireless networks," in Proc. Int. Symp. Inform. Technol., Las Vegas, NV, 2005, pp. 716-722.

[19] P. Svedman, S. K. Wilson, J. Cimini, J. Leonard, and B. E. Ottersten, "A simplified opportunistic feedback and scheduling scheme for OFDM," in Proc. IEEE Veh. Technol. Conf., Spring, Milan, Italy, 2004, vol. 4, pp. 1878-1882.

[20] J. Hämäläinen and R. Wichman, "Performance of multiuser diversity in the presence of feedback errors," in Proc. IEEE Int. Symp. Personal, Indoor and Mobile Commun., Barcelona, Spain, 2004, vol. 1, pp. 599-603.

[21] X. Qin and R. Berry, "Opportunistic splitting algorithms for wireless networks," in Proc. IEEE INFOCOM, Hong Kong, China, 2004, vol. 3, pp. 1662-1672.

[22] H. Cheon, B. Park, and D. Hong, "Adaptive multicarrier system with reduced feedback information in wideband radio channels," in Proc. IEEE Veh. Technol. Conf., Fall, Amsterdam, The Netherlands, 1999, vol. 5, pp. 2880-2884.

[23] T. Lestable and M. Bartelli, "LZW adaptive bit loading," in Proc. IEEE Int. Symp. Advances in Wireless Commun., Victoria, B.C., Canada, Sep. 2002.

[24] T. A. Welch, "A technique for high-performance data compression," IEEE Computer, vol. 17, no. 6, pp. 8-19, 1984.

[25] H. Nguyen and T. Lestable, "Compression of bit loading power vectors for adaptive multi-carrier systems," in Proc. IEEE Int. Midwest Symp. Circuits Syst., Hiroshima, Japan, 2004, pp. 243-246.

[26] J. Capon, "A probabilistic model for run-length coding of pictures," IRE Trans. Inform. Theory, pp. 157-163, 1959.

[27] V. P. G. Jimenez and A. G. Armada, “An adaptive MIMO-OFDM system: Design and performance evaluation," in Proc. Int. Symp. Wireless Commun. Syst., Valencia, Spain, 2006, pp. 810-814.

[28] J. Gross, H.-F. Geerdes, and A. Wolisz, "On the signaling overhead in dynamic OFDMA wireless systems," Jahrestagung der Gesellschaft für Informatik, vol. 35, 2005, in press.

[29] J. Gross, H.-F. Geerdes, H. Karl, and A. Wolisz, "Performance analysis of dynamic OFDMA systems with inband signaling," IEEE J. Sel. Areas Commun., vol. 24, no. 3, pp. 427-436, Mar. 2006.

[30] T. Eriksson and T. Ottosson, "Compression of feedback in adaptive OFDM-based systems using scheduling," IEEE Commun. Lett., 2007. in press.

[31] M. Sternad, T. Svensson, and G. Klang, "WINNER MAC for cellular transmission," in Proc. IST Mobile Summit, Mykonos, Greece, 2006.

[32] A. Narula, M. J. Lopez, M. D. Trott, G. W. Wornell, M. Inc, and M. A. Mansfield "Efficient use of side information in multiple-antenna data transmission over fading channels," IEEE J. Sel. Areas Commun., vol. 16, no. 8, pp. 1423-1436, Oct. 1998.

[33] K. K. Mukkavilli, A. Sabharwal, E. Erkip, and B. Aazhang, "On beamforming with finite rate feedback in multiple-antenna systems," IEEE Trans. Inform. Theory, vol. 49, no. 10, pp. 2562-2579, Oct. 2003.

[34] D. J. Love and R. W. Heath, Jr., "Limited feedback precoding for spatial multiplexing systems," in Proc. IEEE Global Telecommun. Conf., San Francisco, CA, 2003, vol. 4, pp. 1857-1861.

[35] J. C. Roh and B. D. Rao, "An efficient feedback method for MIMO systems with slowly time-varying channels," in Proc. IEEE Wireless Commun. Netw. Conf., Atlanta, GA, 2004, vol. 2, pp. 760-764.

[36] M. Sharif and B. Hassibi, "On the capacity of MIMO broadcast channels with partial side information," IEEE Trans. Inform. Theory, vol. 51, no. 2, pp. 506-522, 2005.

[37] A. Gersho and R. M. Gray, Vector Quantization and Signal Compression. Boston, MA: Kluwer, 1992.

[38] W. Santipach and M. L. Honig, “Asymptotic performance of MIMO wireless channels with limited feedback," in Proc. IEEE Military Commun. Conf., MILCOM 2003, 2003, vol. 1, pp. 141-146.

[39] C. K. Au-Yeung and D. J. Love, "Performance analysis of random vector quantization limited feedback beamforming," in Proc. Asilomar Conf, Signals, Syst. Comput., Pacific Grove, CA, 2005, pp. 408-412.

[40] N. Jindal, "MIMO broadcast channels with finite rate feedback," in Proc. IEEE Global Telecommun. Conf., St. Louis, MO, 2005, pp. 1520-1524.

[41] B. Mielczarek and W. Krzymien, "Flexible channel feedback quantization in multiple antenna systems," in Proc. IEEE Veh. Technol. Conf., Spring, Stockholm, Sweden, 2005, vol. 1, pp. 620-624.

[42] J. C. Roh and B. D. Rao, "Transmit beamforming in multiple-antenna systems with finite rate feedback: A VQ-based approach," IEEE Trans. Inform. Theory, vol. 52, no. 3, pp. 1101-1112, 2006.

[43] D. J. Love, R. W. Heath, Jr., and T. Strohmer, "Grassmannian beamforming for multiple-input multiple-output wireless systems," IEEE Trans. Inform. Theory, vol. 49, no. 10, pp. 2735-2747, Oct. 2003.

[44] M. Kountouris and D. Gesbert, "Robust multi-user opportunistic beamforming for sparse networks," in Proc. IEEE Workshop on Signal Process. Advances in Wireless Commun., New York, 2005, pp. 975-979.

[45] "3GPP TR 25.943 technical specification group radio access networks; deployment aspects," Tech. Rep., 2002.

[46] L. Yang, M. S. Alouini, and D. Gesbert, "Further results on selective multiuser diversity," in Proc. 7th ACM Int. Symp. Modeling, Analy. Simulation of Wireless and Mobile Syst., Venice, Italy, 2004, pp. 25-30.

[47] D. L. Goeckel, “Adaptive coding for time-varying channels using outdated fading estimates," IEEE Trans. Commun., vol. 47, no. 6, pp. 844-855, Jun. 1999.

[48] G. Jöngren and M. Skoglund, "Quantized feedback information in orthogonal space-time block coding," IEEE Trans. Inform. Theory, vol. 50, no. 10, pp. 2473-2486, Oct. 2004. 


\section{ABOUT THE AUTHORS}

Thomas Eriksson was born on April 7, 1964, in Skövde, Sweden. He received the M.Sc. degree in electrical engineering and the Ph.D. degree in information theory from the Chalmers University of Technology, Gothenburg, Sweden, in 1990 and 1996, respectively.

He was with AT\&T Laboratories-Research from 1997 to 1998 , and from 1998 and 1999, he was working on a joint research project with the Royal Institute of Technology and Ericsson Radio Sys-

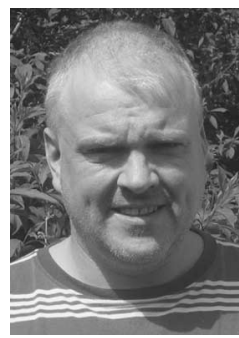

tems AB. In 2003 and 2004, he was a Guest Professor at Yonsei University, Seoul, Korea. Currently, he is an Associate Professor with the Department of Signals and Systems, Chalmers University of Technology. His research interests include communication systems, source coding, and information theory. Specific interests include vector quantization, speaker recognition, multiuser diversity, and system modeling of nonideal hardware components.
Tony Ottosson was born in Uddevalla, Sweden, in 1969. He received the Ph.D. degree from the Department of Information Theory, Chalmers University of Technology, Gothenburg, Sweden, in 1997.

Currently, he is a Professor with the Communication Systems Group, Department of Signals and Systems, Chalmers University of Technology. During 1999, he was also working as a Research Consultant at Ericsson Inc., Research Triangle

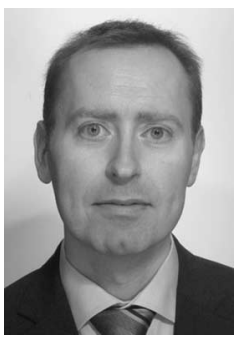
Park, NC. He has published more than 90 journal and conference papers, and holds several patents. His research interests are in communication systems and information theory. Specific topics are modulation, coding, CDMA, multiuser detection, combined source-channel coding, joint decoding techniques, synchronization, cross-layer interaction, multiuser diversity, scheduling, and MIMO systems.

Dr. Ottosson has served as Associate Editor for IEEE TRANSACTIONS ON VeHICULAR TECHNOLOGY from 2000 to 2004 